\title{
State Space Models for Dynamic Style Analysis of Portfolios*
}

\author{
Adrian Pizzinga** \\ Cristiano Fernandes ${ }^{* * *}$
}

\begin{abstract}
This paper presents a framework and methods for the estimation of linear and non-linear state space (SS) models, occasionally subject to restrictions, to construct and estimate several models for style analysis with time varying exposures. The study is conducted by applying these models to an artificial portfolio and to return series of Brazilian investment funds. The results confirm the belief that dynamic allocations in a portfolio are a more realistic assumption for investment funds management.
\end{abstract}

Keywords: Dynamic Style Analysis, State Space Models, Kalman Recursions, Restrictions, Approximate Non-linear Filters.

JEL Codes: C15, C22, G11, G23.

\footnotetext{
${ }^{*}$ Submitted in October 2004. Revised in August 2005. This work was partially supported by CNPq (National Council for Scientific and Technological Development).

${ }^{* *} \mathrm{PhD}$ student in Statistics at the Department of Electrical Engineering of PUC-Rio and CNPq fellowship holder. E-mail: adrianhp@ig.com.br

${ }^{* * *}$ Professor at the Department of Electrical Engineering of PUC-Rio.

E-mail: cris@ele.puc-rio.br
}

Brazilian Review of Econometrics

v. $26, \mathrm{n}^{\circ} 1$, pp. 31-66 May 2006 


\section{Introduction}

Return-based style analysis - or plainly style analysis - (Sharpe (1988), Sharpe (1992), Varga and Valli (1998), Otten and Bams (2000), Brown and Goetzmann (2003) and De Roon et al. (2004)) is basically an investigative process on the composition of an investment fund or portfolio. ${ }^{1}$ Its major aim is to identify the allocations - or exposure - to different financial market niches (stock market, fixed-income securities market, exchange rate market and its respective derivatives, commodities market etc.). A remarkable feature of style analysis is that it is an external investigation, since only "easily" obtained information on investment fund returns and on financial market indicators is taken into consideration.

The rationale behind the style analysis lies in determining the exposure of pension funds to the risk of different asset classes through the purchase of quotas of investment funds open to financial resource mobilization. Figure 1 summarizes this flow for the case of three investment funds and four asset classes. The arrows indicate possible exposures.

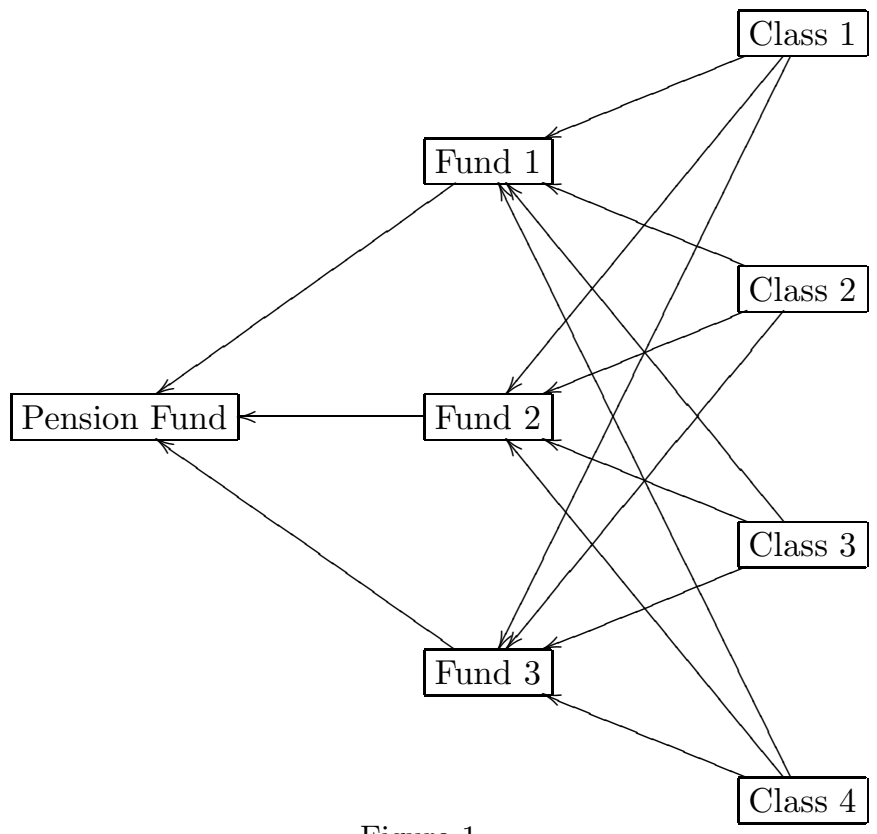

Figure 1

Exposures of a pension fund to different asset classes

\footnotetext{
${ }^{1}$ Occasionally, the term "portfolio" may mean "fund" and vice versa. 
It is crucial to obtain information about these exposures so that new investment strategies can be devised, including also efficient reallocations of the capital invested by the pension fund manager.

Throughout the years, style analysis has taken on other roles, as acknowledged by capital market participants (general investors, banks, asset managements, brokers, risk analysts, organizations that outsource investment analyses, rating firms, regulating agents, among others). These roles include: categorizing investment funds based on a more solid empirical base (see Varga and Valli (1998)); assessing the historical performance of a portfolio; checking whether a given manager of a portfolio open to financial resource mobilization complies with the investment policy he/she promised to investors; and establishing benchmarks for a specific funds market see (Sharpe (1992)).

As observed, style analysis is an important tool in promoting transparency, which is pivotal to the operation and liquidity of the financial market. This paper aims to provide a generalization of this technique, so that portfolio compositions can be dynamically estimated over time, which theoretically allows the identification of possible changes in the management style of the analyzed fund.

The paper is organized as follows. Section 2 revisits the static style analysis. At the end of this section, there are two criticisms, with special attention being given to the lack of dynamic range of the allocations to be estimated. Section 3 describes the major topics related to (linear and non-linear) state space models and their estimation methods. Strategies for the imposition of linear restrictions on the estimation of the state vector are also discussed. Section 4 proposes ways to solve the lack of dynamic range of the original style analysis. These propositions are totally based on the development of space state models for dynamic style analyses. Section 5 assesses the models proposed in the previous section by applying an exercise for the estimation, over time, of an artificial portfolio composition. Section 6 shows applications with real data concerning two Brazilian investment funds. Section 7 concludes and suggests directions for future work.

\section{Static Style Analysis}

\subsection{Asset class factor model}

Originally, (static) style analysis is carried out by adjusting the following asset class factor model (Sharpe $(1988,1992)$ :

$$
R_{t}^{c}=\beta_{1} R_{t 1}+\beta_{2} R_{t 2}+\cdots+\beta_{m} R_{t m}+\varepsilon_{t}, \quad t=1,2, \ldots
$$

In the expression in (1), note that: (i) $R_{t}^{c}$ is the stochastic process that represents a portfolio return at time $t$; (ii) $R_{t 1}, R_{t 2}, \ldots, R_{t m}$ are the stochastic processes that stand for the returns on financial indicators (in Brazil: Ibovespa, CDI (Interbank Deposit Certificate), dollar etc.) associated with $m$ asset classes, which should satisfy the assumptions of exhaustiveness (the model does not lack relevant 
variables), of mutual exclusiveness and of different behavior (there exists no strong multicollinearity); (iii) $\beta_{1}, \beta_{2}, \ldots, \beta_{m}$ are a priori unknown, but estimable, allocations/exposures; and (iv) $\varepsilon_{t}$ is a white noise with variance $\sigma^{2}$. In this context, the error $\varepsilon_{t}$ should be interpreted as part of the return concerning the selection of the manager and imperfections in the attempt of market reproduction by the adopted $m$ asset classes.

Let us consider the following restrictions on exposures $\beta_{1}, \beta_{2}, \ldots, \beta_{m}$ :

$$
\begin{gathered}
\sum_{i=1}^{m} \beta_{i}=1 \\
\beta_{i} \geq 0, i=1,2, \ldots, m
\end{gathered}
$$

Restriction (2) is interpreted as a portfolio constraint. Restriction (3), in its turn, represents the imposition that the portfolio assets are not leveraged or have short positions ${ }^{2}$ in their composition.

According to the taxonomy described in De Roon et al. (2004), it is possible to implement three types of style analysis, depending on which restrictions are being imposed:

- Weak Style Analysis: no restriction is imposed;

- Semi-Strong Style Analysis: restriction (2) is imposed;

- Strong Style Analysis: restrictions (2) and (3) are imposed.

Observe that strong style analysis is only appropriate for funds that are not leveraged and/or for investments in sale derivatives, as pointed out by de De Roon et al. (2004).

\subsection{Implementation}

In static style analysis, estimated allocations should be such that the sum of squares or the sample variance of residuals could be minimized. Considering the sum of squares of the residuals, if no restriction is imposed (i.e., weak style analysis) or if only restriction (2) is adopted (i.e., semi-strong style analysis), then usual methods for the estimation of linear regression models (ordinary least squares and least mean squares, respectively) constitute natural adjustment solutions. However, if both restrictions in (2) and (3) should be imposed simultaneously (i.e., strong style analysis), then more complex optimization methods are necessary. Examples of these methods include the gradient method described in Sharpe (1988) apud Sharpe (1988), Sharpe (1992) and Varga and Valli (1998), Kuhn Tucker's method described in detail by Otten and Bams (2000).

\footnotetext{
${ }^{2}$ In other words, the portfolio is not sold in any asset class.
} 
After the estimated allocations $\widehat{\beta}_{1}, \widehat{\beta}_{2}, \ldots, \widehat{\beta}_{m}$, as well as their respective standard errors for the inference on actual allocations (see Otten and Bams (2000) for the case of strong style analysis), are obtained and properly interpreted, the style analysis will have been implemented.

\subsection{Limitations of style analysis in its original framework}

Static style analysis requires more consistent solutions to two problems that are endogenous to it and which are the major source of criticism. These are, namely:

1. Efficient benchmarks are not always available for the representation of possible asset classes in the financial market.

2. The actual allocations/exposures of a portfolio, even if they are passive, do not always remain the same throughout time, and can be changed by the manager - an idea that is also shared by Swinkels and Van de Sluis (2001).

Statistically speaking, the first problem translates into lack of relevant explanatory variables for a regression model. In the case of the Brazilian funds market, Varga and Valli (1998) highlight that this occurs mainly in the search of benchmarks for fixed-income investments.

The second problem occurs when the asset class factor model, described in (1), establishes that theoretical allocations $\beta_{1}, \beta_{2}, \ldots, \beta_{m}-$ and consequently, estimated allocations $\widehat{\beta}_{1}, \widehat{\beta}_{2}, \ldots, \widehat{\beta}_{m}$ - must be fixed over time. Therefore, one can plausibly suppose that the original proposition for the style analysis, static by construction, is not appropriate to mathematically represent/approximate what actually happens over time to the allocations made by the manager.

Section 4 presents three solutions to the second problem, one to each type of style analysis (weak, semi-strong and strong), followed by implementation strategies totally based on state space models, which are to be dealt with in the subsequent section.

\section{State Space Models}

\subsection{Linear models, Kalman recursions and imposition of restrictions}

A linear Gaussian state space (SS) model for a discrete-time $p$-variate observable stochastic process $Y_{t}=\left(Y_{t 1}, Y_{t 2}, \ldots, Y_{t p}\right)^{\prime}$, defined in an appropriate probability space $(\Omega, \Im, P)$, is described by the following set of equations: 


$$
\begin{aligned}
Y_{t} & =Z_{t} \alpha_{t}+d_{t}+\varepsilon_{t} \\
\alpha_{t+1} & =T_{t} \alpha_{t}+c_{t}+R_{t} \eta_{t} \\
t & =1,2, \ldots
\end{aligned}
$$

The first equation is known as measurement equation (or, alternatively, as observation equation), whereas the second one is the state equation. The following assumptions and nomenclatures exist: (i) $\varepsilon_{t}$ and $\eta_{t}$ are Gaussian stochastic process with zero mean, $p$-variate and $r$-variate, respectively, non-observable and independent of each other over time, with defined non-negative covariance matrices $\operatorname{var}\left(\varepsilon_{t}\right)=H_{t}$ and $\operatorname{var}\left(\eta_{t}\right)=Q_{t}$; (ii) the non-observable $m$-variate $\alpha_{t}$ process is called state; (iii) $Z_{t}, d_{t}, H_{t}, T_{t}, c_{t}, R_{t}$ and $Q_{t}$ are called system matrices and behave deterministically over time. Detailed examples of SS models can be obtained in Harvey (1989), Brockwell and Davis (1996), Tanizaki (1996), and, more recently, in Durbin and Koopman (2001).

For linear models, Kalman filtering, prediction and smoothing theory, properly combined with (quasi-) maximum likelihood estimation of unknown parameters, provides the most natural, most elegant, most computationally efficient, and according to the minimization of the mean of the squared error, the most efficacious alternative for the estimation of state vector $\alpha_{t}$ over time. Before presenting these recursions, the following basic notation is necessary:

- $n$ is the size of a series extracted from the process $Y_{t}$;

- $\mathcal{F}_{j}$ is the $\sigma$-algebra produced by univariate measurements up to time $j$, i.e., $\mathcal{F}_{j} \equiv \sigma\left(Y_{11}, \ldots, Y_{1 p}, \ldots, Y_{j 1}, \ldots, Y_{j p}\right)$;

- $a_{t \mid j} \equiv E\left(\alpha_{t} \mid \mathcal{F}_{j}\right)$;

- $P_{t \mid j} \equiv E\left[\left(\alpha_{t}-a_{t \mid j}\right)\left(\alpha_{t}-a_{t \mid j}\right)^{\prime} \mid \mathcal{F}_{j}\right]$

- $a_{t} \equiv a_{t \mid t-1}$ and $P_{t} \equiv P_{t \mid t-1}$;

- $\hat{\alpha}_{t} \equiv a_{t \mid n}$ and $V_{t} \equiv P_{t \mid n}$;

- $v_{t} \equiv Y_{t}-E\left(Y_{t} \mid \mathcal{F}_{t-1}\right)=Y_{t}-Z_{t} a_{t}-d_{t}$ is the innovation vector; and

- $F_{t} \equiv E\left(v_{t} v_{t}^{\prime} \mid \mathcal{F}_{t-1}\right)=Z_{t} P_{t} Z_{t}^{\prime}+H_{t}$.

Kalman recursions provide the conditional moments described above for $j=$ $t-1, j=t$ and $j=n$. These recursions are presented next. Their derivations can be found, for instance, in Durbin and Koopman (2001), passim:

- Predicting equations

$$
\begin{aligned}
a_{t+1} & =T_{t} a_{t \mid t}+c_{t} \\
P_{t+1} & =T_{t} P_{t \mid t} T_{t}^{\prime}+R_{t} Q_{t} R_{t}^{\prime}
\end{aligned}
$$


- Updating or filtering equations (the Kalman Filter)

$$
\begin{aligned}
a_{t \mid t} & =a_{t}+P_{t} Z_{t}^{\prime} F_{t}^{-1} v_{t} \\
P_{t \mid t} & =P_{t}-P_{t} Z_{t}^{\prime} F_{t}^{-1} Z_{t} P_{t}
\end{aligned}
$$

- Smoothing equations

$$
\begin{aligned}
\hat{\alpha}_{t} & =a_{t}+P_{t} r_{t-1} \\
r_{t-1} & =Z_{t}^{\prime} F_{t}^{-1} v_{t}+\left(T_{t}-T_{t} P_{t} Z_{t}^{\prime} F_{t}^{-1} Z_{t}\right)^{\prime} r_{t} \\
V_{t} & =P_{t}-P_{t} N_{t-1} P_{t} \\
N_{t-1} & =Z_{t}^{\prime} F_{t}^{-1} Z_{t}+\left(T_{t}-T_{t} P_{t} Z_{t}^{\prime} F_{t}^{-1} Z_{t}\right)^{\prime} N_{t}\left(T_{t}-T_{t} P_{t} Z_{t}^{\prime} F_{t}^{-1} Z_{t}\right) \\
r_{n} & =0 \text { and } V_{n}=0
\end{aligned}
$$

If, by chance, $F_{t}$ is singular throughout the recursions, a generalized inverse equation may be used, which will not change the fact that conditional moments are singular $P$-almost surely. Also note that, outside the normality assumption, these equations still make sense and should be understood as orthogonal projections and respective mean squared errors. For further and more general information, see Brockwell and Davis (1996) (updating equations), de De Jong (1989) (smoothing equations) and, for an abridged discussion, see Pizzinga (2004), chapter 3.

Occasionally, the expression in (4) might not be the best option for a given practical situation. As a matter of fact, the process $\alpha_{t}$, depending on the problem at hand, may endogenously satisfy restrictions of the type

$$
A_{t} \alpha_{t}=q_{t}
$$

where $A_{t}$ is a matrix $k \times m$ and $q_{t}$ is a vector, probably a random one, with dimension $k \times 1$. Note that the restrictions described in (8) may vary over time, both in terms of type and in terms of number.

After this additional information, it would be reasonable to include it in the estimation of the state vector using the previously described Kalman recursions. Actually, this issue was the major focus of the study conducted by Doran (1992) and has been revisited by Pizzinga (2004) and Pizzinga et al. (2006a), basically including unexplored theoretical aspects. In short, the strategy that guarantees the transfer of restrictions in (8) to Kalman updating and smoothing equations is presented next, as a theorem, whose demonstration can be found in Pizzinga et al. (2006a).

Theorem 1 If measurement vectors $Y_{t}$ are replaced by $Y_{t}^{*}=\left(Y_{t}^{\prime}, q_{t}^{\prime}\right)^{\prime}$, matrices $Z_{t}$ are replaced by $Z_{t}^{*}=\left[\begin{array}{ll}Z_{t}^{\prime} & A_{t}^{\prime}\end{array}\right]^{\prime}$, vectors $d_{t}$ are replaced by $d_{t}^{*}=\left(d_{t}^{\prime}, 0^{\prime}\right)^{\prime}$, and error vectors $\varepsilon_{t}$ are replaced by $\varepsilon_{t}^{*}=\left(\varepsilon_{t}^{\prime}, 0^{\prime}\right)^{\prime}$, then the Kalman updating and smoothing 
equations applied to this new linear SS model will satisfy the same restrictions in (8), i.e.,

$$
A_{t} a_{t \mid t}=q_{t} \text { and } A_{t} \hat{\alpha}_{t}=q_{t}
$$

So, an augmented (but still linear) state space model should be adopted and Kalman recursions should be applied in the usual manner. The imposition of linear restrictions is being discussed here, but it will be addressed again in the next section as well.

One should highlight that, in practice, system matrices include the estimation of unknown parameters. By grouping all unknown parameters of the model described in (4) into a vector $\psi$, and considering that it may take on values in a parametric space $\Theta$, one then builds an exact log-likelihood function - assessed in a particular $\psi$ - using usual amounts of Kalman filter and amounts obtained from the exact initialization:

$$
\log L(\psi)=-\frac{n p}{2} \log (2 \pi)-\frac{1}{2} \sum_{t=1}^{d} \omega_{t}-\frac{1}{2} \sum_{t=d+1}^{n}\left(\log \left|F_{t}\right|+v_{t}^{\prime} F_{t}^{-1} v_{t}\right)
$$

where

$$
\begin{aligned}
\omega_{t} & =\log \left|F_{\infty, t}\right|, \text { se } F_{\infty, t}>0 \\
& =\log \left|F_{*, t}\right|+v_{t}^{\prime(0)} F_{*, t}^{-1} v_{t}^{(0)}, \text { if } F_{\infty, t}=0
\end{aligned}
$$

The appropriate expressions for $F_{\infty, t}, F_{*, t}$ e $v_{t}^{(0)}$, regarding the exact initial Kalman filter, are described in chapter 5 of Durbin and Koopman (2001). The maximum likelihood estimator of $\psi$ is defined by $\hat{\psi} \equiv \arg \max _{\psi \in \Theta} \log L(\psi)$. When the normality assumption is violated, the expression of (10) should be interpreted as a quasi log-likelihood function and $\hat{\psi}$, in its turn, as a quasi-maximum likelihood estimator.

For recent bibliography on other methods for the adjustment of linear models, see Durbin and Koopman (2001).

\subsection{Non-linear models and approximate nonlinear filters}

In this subsection, we present the expressions to be adopted in this paper for a Gaussian non-linear SS model:

$$
\begin{aligned}
Y_{t} & =Z_{t}\left(\alpha_{t}\right)+\varepsilon_{t} \\
\alpha_{t+1} & =T_{t}\left(\alpha_{t}\right)+R_{t} \eta_{t} \\
t & =1,2, \ldots
\end{aligned}
$$


All previous assumptions about the linear state space model are still valid, except for the fact that $Z_{t}$ and $T_{t}$, which were matrices representing linear transformations, can now constitute any Borel measurable vector transformations. Detailed examples of non-linear state space models can be obtained in Anderson and Moore (1979), Harvey (1989), Fuhrer (1992), Tanizaki (1996) and Pizzinga (2004).

As these are non-linear models, there are some new challenges facing their adjustment and the estimation of the state vector: except for some special cases, it is not possible to obtain closed analytical expressions for the conditional moments of $\alpha_{t}$. This paper will touch upon two approximate methods to estimate such models: the extended Kalman filter and Monte Carlo simulation filter, both combined with the approximate maximum likelihood estimation of unknown parameters. The literature in this field is very extensive and has developed quickly in the past 10 years thanks to the rapid improvement of computational technology. The common element of exact methods for the estimation of non-linear state space models is the use of simulation techniques for the conditional distribution of the state - or of some Borel measurable transformation - given a set of observations, as well as corresponding moments of interest (basic notation in subsection 3.1). By and large, these references can be categorized into four major classes: Markov Chain Monte Carlo (MCMC) - represented by Shephard (1994), Shephard and Pitt (1997) and Gamerman (1998); non-linear filters by Tanizaki and Mariano - Tanizaki and Mariano (1996); method of Durbin and Koopman - Durbin and Koopman (2001); and particle filters and their extensions - represented by the collection edited by Doucet et al. (2001).

The extended Kalman filter (Anderson and Moore (1979), Harvey (1989), Fuhrer (1992), Tanizaki (1996) and Pizzinga (2004)) consists of a heuristic procedure, which aims to establish a parallel with the previously described Kalman predicting and updating recursions, for a model linearized by first-order Taylor expansion. To do that, it is necessary that functions $Z_{t}$ and $T_{t}$ be first-order differentiable.

Considering the previous notation, one can describe the technique using the following steps:

- For a given time $t$, approximate functions $Z_{t}$ and $T_{t}$ in first-order Taylor expansion around $a_{t}$ and $a_{t \mid t}$, respectively. The latter have the same meaning as they did before. Then we have

$$
\begin{aligned}
& Z_{t}\left(\alpha_{t}\right) \cong Z_{t}\left(a_{t}\right)+\dot{Z}_{t}\left(\alpha_{t}-a_{t}\right) \\
& T_{t}\left(\alpha_{t}\right) \cong T_{t}\left(a_{t \mid t}\right)+\dot{T}_{t}\left(\alpha_{t}-a_{t \mid t}\right)
\end{aligned}
$$

where $\dot{Z}_{t}$ is the Jacobian matrix of function $Z_{t}$ assessed in $a_{t}$ and $\dot{T}_{t}$ is the Jacobian matrix of function $T_{t}$ assessed in $a_{t \mid t}$.

- Establish the following linear approximation for the non-linear model, given 
by

$$
\begin{aligned}
Y_{t} & =\dot{Z}_{t} \alpha_{t}+d_{t}+\tilde{\varepsilon}_{t} \\
\alpha_{t+1} & =\dot{T}_{t} \alpha_{t}+c_{t}+R_{t} \tilde{\eta}_{t}
\end{aligned}
$$

where $d_{t}=Z_{t}\left(a_{t}\right)-\dot{Z}_{t} a_{t}, c_{t}=T_{t}\left(a_{t \mid t}\right)-\dot{T}_{t} a_{t \mid t}$ and the new errors $\tilde{\varepsilon_{t}}$ and $\tilde{\eta}_{t}$ are given by the sum of original errors $\varepsilon_{t}$ and $R_{t} \eta_{t}$ with Taylor expansion residuals, which depend on $\alpha_{t}$ and on past measurements up to time $t-1$.

- To the linear approximation given in the previous step, apply the traditional Kalman recursions given in (6) and (5), with the following changes to the filtering and prediction state equations, given by,

$$
\begin{aligned}
a_{t \mid t} & =a_{t}+P_{t} \dot{Z}_{t}^{\prime} F_{t}^{-1}\left(Y_{t}-Z_{t}\left(a_{t}\right)\right) \\
a_{t+1} & =T_{t}\left(a_{t \mid t}\right)
\end{aligned}
$$

Note that: (i) under Gaussian assumption, the quantities computed by the extended Kalman filter can be interpreted as approximations of mean vectors and conditional covariance matrices; (ii) the non-linearity of the original specification is recovered in the definition of pseudo-innovation $\tilde{v}_{t}=Y_{t}-Z_{t}\left(a_{t}\right)$ and in the prediction state equation; and (iii) quantities $d_{t}$ and $c_{t}$ are never calculated, as relevantly highlighted in Harvey (1989:162). For further information about this set of recursions, including a discussion on its advantages and disadvantages, see the previously mentioned references.

From an operational standpoint, the main advantage of the extended Kalman filter over the previously mentioned exact methods is that it is computationally easy to implement. On the other hand, as it is an approximation method, its estimations of the state may turn out to be biased. However, Pizzinga et al. (2006b), after a more extensive investigation into the extended Kalman filter, the Monte Carlo simulation filter and a variant of the method proposed by Durbin and Koopman, concluded that the extended Kalman filter had a similar performance to that of the other two methods, when applied to the non-linear SS model to be discussed in section 4 (see (18) and (21) below).

Monte Carlo simulation filter (Tanizaki (1996) and Pizzinga (2004)), according to the previously cited method, has the aim to improve the previously mentioned extended Kalman filter, in the sense of better calculating (say "approximate") some conditional expectations through stochastic integration by Monte Carlo simulations. The second advantage of this approximate non-linear filter over the previous one is that the first-order differentiability condition of functions $Z_{t}$ and $T_{t}$ is no longer necessary - neither is the continuity condition. Nevertheless, there is a disadvantage in relation to the extended Kalman filter, characterized by the necessary computational effort, as will be explained further ahead.

Before dealing with this approximate filter, add the following definition to the set of previous notations: 
- $Y_{t \mid t-1}$ is the conditional expectation of $Y_{t}$, given the $\sigma$-algebra produced by the past measurements up to time $t-1$, i.e., $Y_{t \mid t-1} \equiv E\left(Y_{t} \mid \mathcal{F}_{t-1}\right)$; and

- $M_{t \mid t-1}$ is the conditional covariance matrix between $Y_{t}$ and $\alpha_{t}$, given the $\sigma$ algebra produced by the past measurements up to time $t-1$, i.e., $M_{t \mid t-1} \equiv$ $E\left[\left(Y_{t}-Y_{t \mid t-1}\right)\left(\alpha_{t}-a_{t}\right)^{\prime} \mid \mathcal{F}_{t-1}\right]$.

The rationale behind Monte Carlo simulation filter is to allow the calculation (say, estimation) of $a_{t \mid t}, P_{t \mid t}, a_{t}, M_{t \mid t-1}, F_{t}, Y_{t \mid t-1}$ and $P_{t}$ with as little bias as possible, which, by construction, cannot be so easily obtained through the extended Kalman filter (see a discussion about this issue in Pizzinga (2004:53$55)$ ).

The Monte Carlo simulation filter recursions are given by:

$$
\begin{aligned}
\hat{Y}_{t \mid t-1} & =\frac{1}{K} \sum_{i=1}^{K}\left(Z_{t}\left(\alpha_{i, t}\right)+\varepsilon_{i, t}\right) \\
\hat{F}_{t} & =\frac{1}{K} \sum_{i=1}^{K}\left(\left(Z_{t}\left(\alpha_{i, t}\right)+\varepsilon_{i, t}\right)-\hat{Y}_{t \mid t-1}\right)\left(\left(Z_{t}\left(\alpha_{i, t}\right)+\varepsilon_{i, t}\right)-\hat{Y}_{t \mid t-1}\right)^{\prime} \\
\hat{M}_{t \mid t-1} & =\frac{1}{K} \sum_{i=1}^{K}\left(\left(Z_{t}\left(\alpha_{i, t}\right)+\varepsilon_{i, t}\right)-\hat{Y}_{t \mid t-1}\right)\left(\alpha_{i, t}-\hat{a}_{t}\right)^{\prime}
\end{aligned}
$$

where the vectors $\mathrm{n}\left(\alpha_{i, t}^{\prime}, \varepsilon_{i, t}^{\prime}\right)$ are independently drawn from

and

$$
N\left(\left(\begin{array}{c}
\hat{a}_{t} \\
0
\end{array}\right),\left(\begin{array}{cc}
\hat{P}_{t} & 0 \\
0 & H_{t}
\end{array}\right)\right), \forall i=1, \ldots, K
$$

$$
\begin{aligned}
& \hat{a}_{t \mid t}=\hat{a}_{t}+\hat{M}_{t \mid t-1}^{\prime} \hat{F}_{t}^{-1}\left(Y_{t}-\hat{Y}_{t \mid t-1}\right) \\
& \hat{P}_{t \mid t}=\hat{P}_{t}-\hat{M}_{t \mid t-1}^{\prime} \hat{F}_{t}^{-1} \hat{M}_{t \mid t-1} \\
& \hat{a}_{t+1}=\frac{1}{K} \sum_{i=1}^{K}\left(T_{t}\left(\alpha_{i, t \mid t}\right)+R_{t} \eta_{i, t}\right) \\
& \hat{P}_{t+1}=\frac{1}{K} \sum_{i=1}^{K}\left(\left(T_{t}\left(\alpha_{i, t \mid t}\right)+R_{t} \eta_{i, t}\right)-\hat{a}_{t+1}\right)\left(\left(T_{t}\left(\alpha_{i, t \mid t}\right)+R_{t} \eta_{i, t}\right)-\hat{a}_{t+1}\right)^{\prime}
\end{aligned}
$$

where the vectors $\left(\alpha_{i, t \mid t}^{\prime}, \eta_{i, t}^{\prime}\right)$ are independently drawn from

$$
N\left(\left(\begin{array}{c}
\hat{a}_{t \mid t} \\
0
\end{array}\right),\left(\begin{array}{cc}
\hat{P}_{t \mid t} & 0 \\
0 & Q_{t}
\end{array}\right)\right), \forall i=1, \ldots, K .
$$


In the recursions above, $K$ is a "sufficiently large" 3 natural number such that sample means take on values relatively close to those of theoretical means. According to the Strong Law of Large Numbers, under the integrability of the random vectors involved, the sequences of sample means converge almost surely towards the actual means as $K$ tends to infinity. Therefore, $K$-asymptotically, the Monte Carlo simulation filter is an "optimal" estimator of the state vector - both in terms of one-step forward prediction and in terms of filtering. For further details about the Monte Carlo simulation filter, also see Tanizaki (1996) and Pizzinga (2004).

To conclude the discussion about these two approximate non-linear filters, it is necessary to discuss the estimation of unknown parameters. Again, denote $\psi$ as the vector for unknown parameters and $\Theta$ as the respective parametric space. Although it is theoretically difficult to establish a "correct" log likelihood for $\psi$ and for the series in question, there exists a mathematically informal alternative for the estimation of $\psi$. This alternative consists of the approximate maximum likelihood, proposed, for instance, in Fuhrer (1992), Tanizaki (1996) and Tanizaki and Mariano (1996). This involves the approximation of actual innovations and their respective conditional covariance matrices by expressions derived from approximate non-linear filters, producing an approximate log likelihood function. For example, if the extended Kalman filter is chosen for the recursive estimation of the state, the approximate log likelihood function will be given by the expression in (10), including the following expressions:

$$
\begin{aligned}
\tilde{v}_{t} & =Y_{t}-Z_{t}\left(a_{t}\right) \\
F_{t} & =\dot{Z}_{t} P_{t} \dot{Z}_{t}^{\prime}+H_{t}
\end{aligned}
$$

where $\dot{Z}_{t}$ and $a_{t}$ are defined in (12) and in (14). Completely analogous constructions for the Monte Carlo simulation filter may be established. The estimate resulting from the numerical maximization of the approximate log likelihood function is known as approximate maximum likelihood estimate.

\section{State Space Models for Dynamic Style Analyses}

\subsection{State equation}

An interesting way to deal with the second problem regarding the traditional style analysis - lack of dynamics of the allocations/exposures to be estimated would be the use of state space models.

Initially, one builds the state equation, which, due to its ease of use and appropriateness for practical problems, will have a linear and Gaussian distribution

\footnotetext{
${ }^{3}$ Occasionally, the second expression of (16) regarding $\hat{P}_{t \mid t}$ can produce a definite negative matrix! The explanation for such problem is that $\hat{F}_{t}$ and $\hat{M}_{t \mid t-1}$ stem from simulations, which may cause poor approximation of the real matrices. Therefore, in practical situations, $K$ may have to be really "very large".
} 
with time-invariant matrices, regardless of the type of style analysis selected. This would then be given by

$$
\beta_{t+1}=\beta_{t}+\eta_{t}, \eta_{t} \sim N I D(0, Q)
$$

where $Q$ may be diagonal. With this proposal of a Gaussian vector random walk, parameter estimation becomes much simpler and much more stable. Two are the reasons that can corroborate the specification proposed in (18):

- Parsimony and simplicity, since there are no theoretical signs for the selection of a more complex specification and, consequently, one that is statistically more difficult to be dealt with; and

- Accommodation of exposures that may go through fundamental changes over time, due to nonstationary behavior.

\subsection{Measurement equations}

The measurement equation, which will describe the behavior of portfolio return, will depend upon the type of analysis selected. As there are three possibilities, there are three equations, one for each situation, to be combined with the state equation given in (18). By denoting $R_{t}=\left(R_{t 1}, R_{t 2}, \ldots, R_{t m}\right)^{\prime}$, the equations are:

- Weak Style Analysis:

$$
R_{t}^{c}=R_{t}^{\prime} \beta_{t}+\varepsilon_{t}, \varepsilon_{t} \sim N I D\left(0, \sigma^{2}\right)
$$

In this case, the resultant state space model is linear and univariate, thus characterizing the nature of a weak style analysis. Evidently, $Y_{t}=R_{t}^{c}$, $Z_{t}=R_{t}^{\prime}, d_{t}=0$ and $H_{t}=\sigma^{2}$, for all time $t$ (see equations in (4)). This model, with less than one intercept term and with diagonal $Q$ in the state equation in (18), is the same one suggested in expressions (4), (5) and (6) by Swinkels and Van de Sluis (2001).

- Semi-Strong Style Analysis

$$
\begin{aligned}
\left(\begin{array}{c}
R_{t}^{c} \\
1
\end{array}\right) & =\left(\begin{array}{c}
R_{t}^{\prime} \\
1 \cdots 1
\end{array}\right) \beta_{t}+\left(\begin{array}{c}
\varepsilon_{t} \\
0
\end{array}\right), \quad\left(\begin{array}{c}
\varepsilon_{t} \\
0
\end{array}\right) \\
& \sim \operatorname{NID}\left(\left(\begin{array}{l}
0 \\
0
\end{array}\right),\left(\begin{array}{cc}
\sigma^{2} & 0 \\
0 & 0
\end{array}\right)\right)
\end{aligned}
$$

Now, the resultant state space model is still linear, but bivariate, due to the increase in the measurement vectors such that a linear restriction is imposed on the state vector, which determines that all coefficients must sum 1 (one) 
for all time $t-\mathrm{a}$ restriction that stems from the semi-strong style analysis proposal. According to Theorem 1 in subsection 3.1, the adjustment of this model with all the tools involving Kalman recursions, still combined with the (quasi-) maximum likelihood methods, will guarantee that state vectors satisfy the imposed portfolio restriction for all time $t$. Note that the system matrices given by the equations in (4) are determined in an obvious manner. For example, $\mathrm{Z}_{\mathrm{t}} \equiv\left(\begin{array}{c}\mathrm{R}_{\mathrm{t}}{ }^{\prime} \\ 1 \ldots 1\end{array}\right)$, for all $t$.

- Strong Style Analysis:

$$
R_{t}^{c}=\omega_{t 1} R_{t 1}+\omega_{t 2} R_{t 2}+\ldots+\omega_{t m} R_{t m}+\varepsilon_{t}, \varepsilon_{t} \sim N I D\left(0, \sigma^{2}\right)
$$

where

$$
\omega_{t j}=\omega_{t j}\left(\beta_{t}\right) \equiv \frac{\exp \left(\beta_{t j}\right)}{\sum_{i=1}^{m} \exp \left(\beta_{t i}\right)}, j=1, \ldots, m
$$

In this framework, the resultant state space model is non-linear (in the measurement equation only) and univariate, incorporating both restrictions of a strong style analysis, i.e., the allocations (now represented by $\omega_{t 1}, \omega_{t 2}$, $\ldots, \omega_{t m}$ ) sum 1 (one) and are non-negative for all $t$. To adjust this model, it has been suggested that the approximate non-linear filters discussed in subsection 3.2 (extended Kalman filter and Monte Carlo simulation filter), along with the approximate maximum likelihood method, be used. It should be highlighted that $Z_{t}\left(\beta_{t}\right)=\omega_{t 1} R_{t 1}+\omega_{t 2} R_{t 2}+\ldots+\omega_{t m} R_{t m}$ and $T_{t}\left(\beta_{t}\right)=$ $\beta_{t}$, where $Z_{t}$ and $T_{t}$ are the functions given in the specification in (11), which, as can be directly observed, are in agreement with the differentiability assumption of all orders.

One has therefore three types of Dynamic Style Analysis (DSA), whose associated models can be called asset class dynamic factor models. DSA, just as in its previously discussed traditional static version (SSA), can be weak, semi-strong, or strong. The adjustment of the asset class dynamic factor model depends on which DSA is chosen. Table 1 summarizes the three types of models. 
Table 1

SS models proposed for each type of dynamic style analysis

\begin{tabular}{l|c|l}
\hline $\begin{array}{c}\text { Dynamic style } \\
\text { analysis }\end{array}$ & $\begin{array}{c}\text { SS model } \\
\text { (measurement equation) }\end{array}$ & \multicolumn{1}{c}{$\begin{array}{c}\text { Adjustments } \\
\text { proposed }\end{array}$} \\
\hline Weak & $(19)$ & $\begin{array}{l}\text { Kalman recursions } \\
\text { w/ maximum likelihood }\end{array}$ \\
\hline Semi-strong & $(20)$ & $\begin{array}{l}\text { Kalman recursions } \\
\text { w/ maximum likelihood }\end{array}$ \\
\hline Strong & $(21)$ & $\begin{array}{l}\text { Approximate non-linear filters } \\
\text { w/ approximate maximum likelihood }\end{array}$ \\
\hline
\end{tabular}

\section{Analysis of an Artificial Portfolio}

This section aims to illustrate the DSA discussed in the previous section by applying it to an artificial portfolio, whose returns and allocations were produced according to some specific and known rule. This exercise provides a satisfactory way to check the efficacy of the technique in a simulated environment and to compare different proposals for DSA (weak, semi-strong and strong). The market indicators analyzed in this study (as well as the ones that will be discussed in the subsequent section, regarding Brazilian fund portfolios), are: CDI, Dollar, IBOVESPA, IGP-M (General Market Price Index), Quantum Cambial and Quantum Fixed Income. The latter two represent the Dollar exchange rate and fixed-income derivative markets, respectively, and can be obtained by the Quantum Axis system. ${ }^{4}$

All implementations (in this section and in the subsequent ones) were made using Ox 3.0 (Doornick, 2001) with occasional use of SsfPack 3.0 (Koopman et al., 2002). ${ }^{5}$

The artificial portfolio had its actual allocation distributed among the CDI, IBOVESPA and Quantum Cambial indicators, according to the following description: 6

- From the first week in January 2001 to the second week in April 2001, the allocations among indicators were as follows: 30\% CDI, 20\% IBOVESPA and 50\% Quantum Cambial;

- From the third week in April 2001 to the third week in December 2001, the allocations were as follows: $100 \%$ CDI and 0\% for the other indicators;

- From the fourth week in December 2001 to the second week in April 2002, the allocations were as follows: 13\% CDI, 70\% IBOVESPA and 17\% Quantum Cambial;

\footnotetext{
${ }^{4}$ www.quantumfundos.com.br.

${ }^{5}$ An Athlon XP 2200 Mhz with 378 MB-RAM was used.

${ }^{6}$ This portfolio follows the same type of logic used in the simulations by Swinkels and Van de Sluis (2001). However, here, a larger number of indicators was used and the changes in actual allocations are much more "aggressive"; thus, the portfolio here is theoretically "more difficult" to be estimated.
} 
- From the third week in April 2002 to the third week in June 2002, the allocations were as follows: 13\% CDI, 40\% IBOVESPA and 47\% Quantum Cambial; and

- From the fourth week in June 2002 to the fourth week in December 2002, the allocations were as follows: 100\% Quantum Cambial and 0\% for the other indicators.

Weekly allocation series were then constructed and, consequently, a corresponding weekly return series was also built for 2001 and 2002 (total of 104 observations). Based on the return series, of the three indicators used in the distribution of allocations and in another two indicators - Dollar and IGP-M - we adjusted the three models proposed in the previous section. For the first and second models, which are linear, smoothed allocations were calculated using the Kalman filter. The third model, which is non-linear and, as discussed, has the perspective of a strong DSA, was estimated using the extended Kalman filter and the Monte Carlo simulation filter, which produced filtered allocations. Next, we show the graphs on which the estimated allocations (smoothed or filtered, depending on the type of adjustment) were compared with actual allocations for each of the adjustments. Note that $95 \%$ confidence intervals are only presented for weak and semi-strong DSAs, since the current formats of the extended Kalman filter and of the Monte Carlo simulation filter do not allow yet for measurements on precision. 

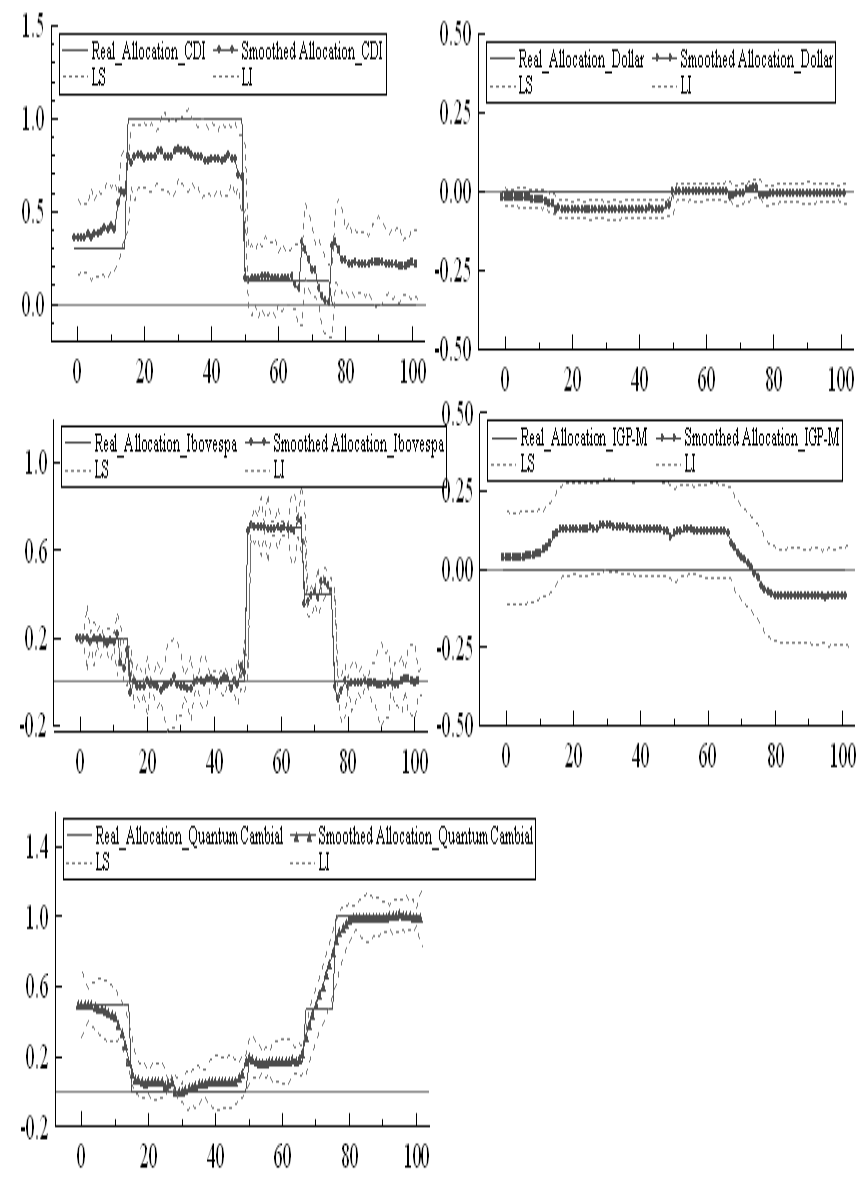

Figure 2

Weak dynamic style analysis of an artificial portfolio 

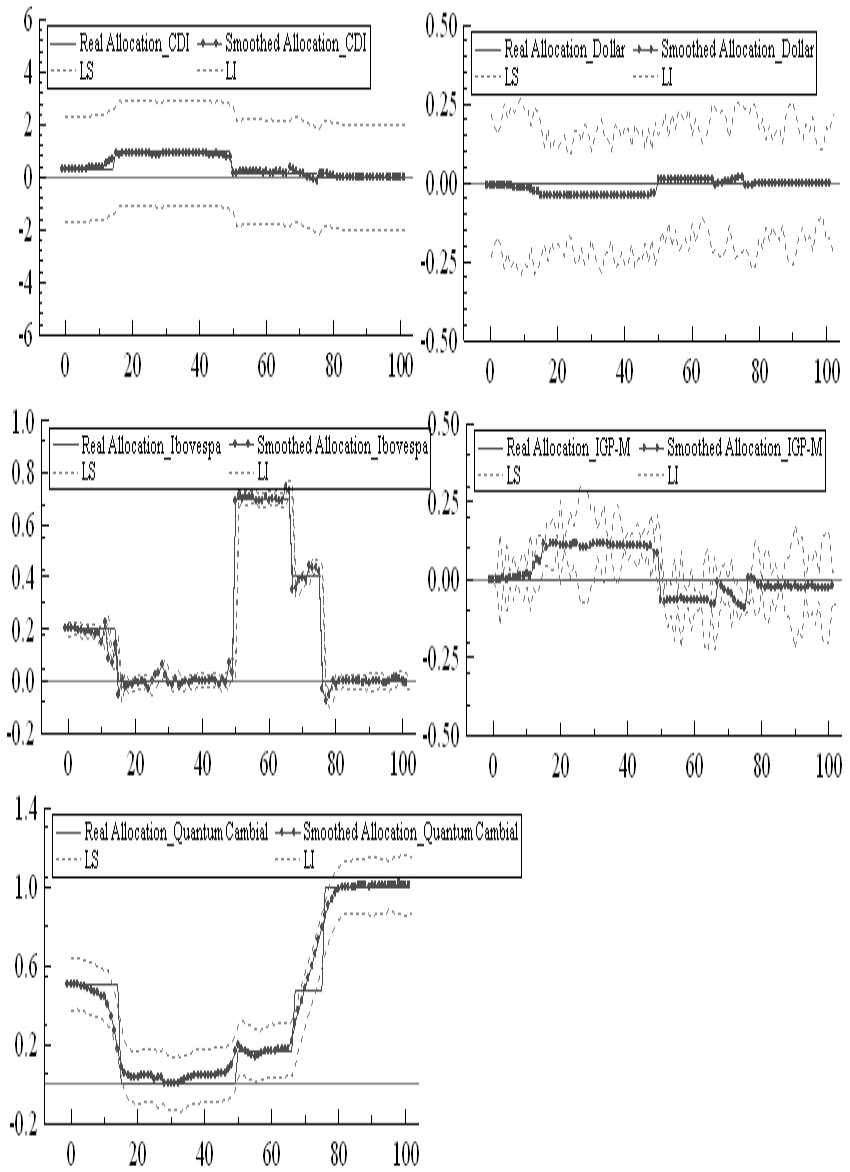

Figure 3

Semi-strong dynamic style analysis of an artificial portfolio 


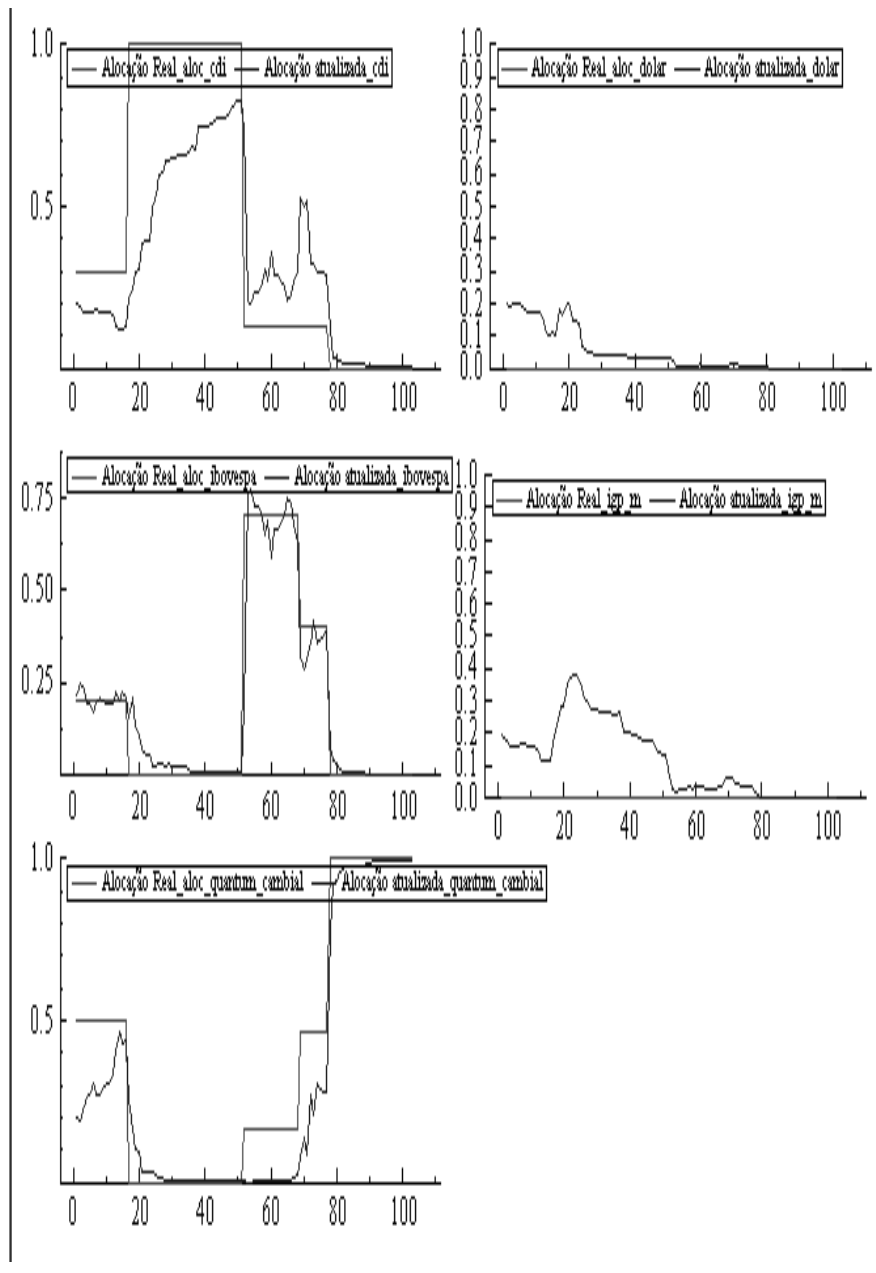

Figure 4

Strong dynamic style analysis of an artificial portfolio using the extended Kalman filter 

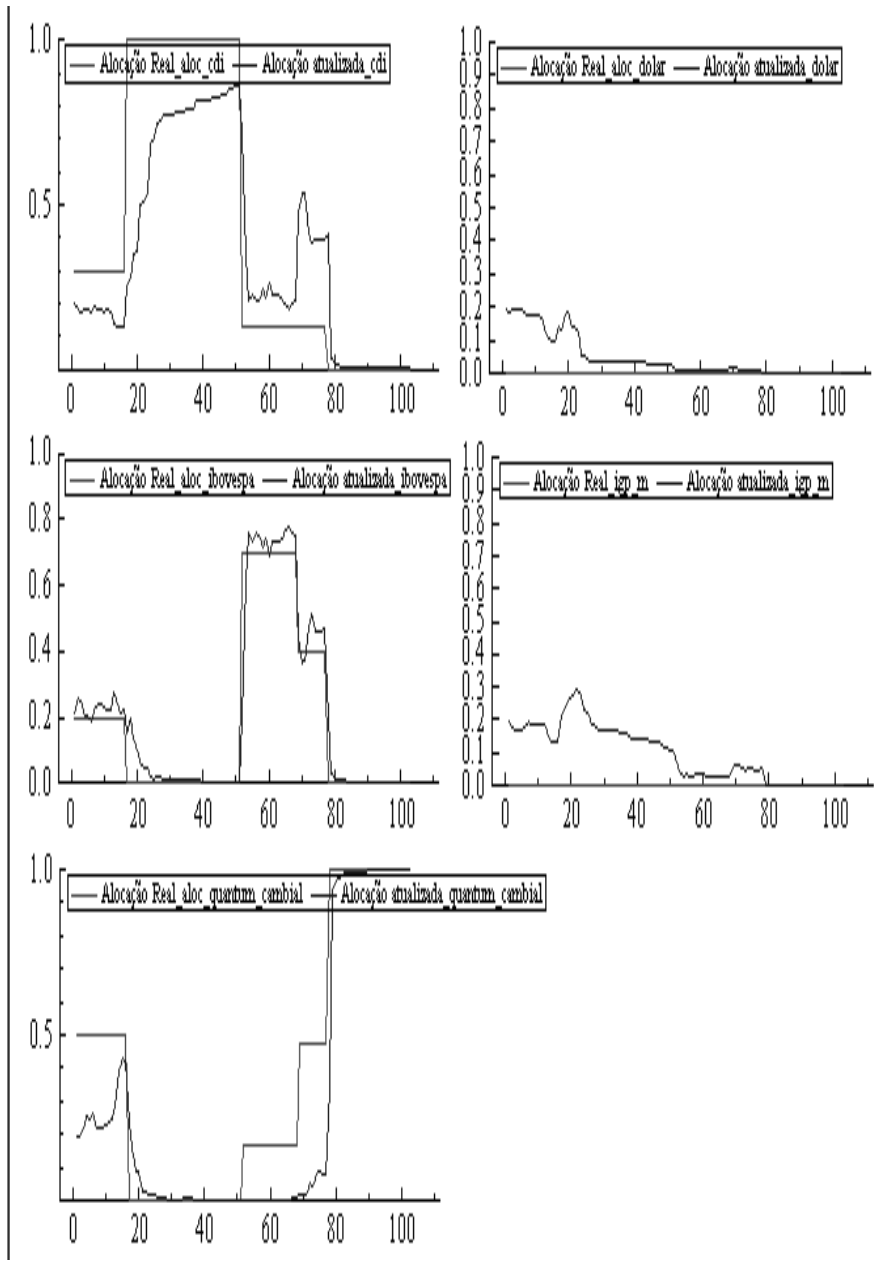

Figure 5

Strong dynamic style analysis of an artificial portfolio using the Monte Carlo simulation filter

By carefully examining figures $2,3,4$ and 5 , one can observe that the artificial portfolio was satisfactorily "recovered" by the three possible DSAs, with virtually total pertinence over time of actual allocations to confidence intervals regarding weak and semi-strong style analyses. One can also observe that Dollar and IGP$\mathrm{M}$, which are nothing but two irrelevant explanatory variables here, had their corresponding allocations estimated close to zero, throughout time. This was more 
efficiently achieved by weak and semi-strong DSAs, especially at the beginning of the series. Finally, despite the sudden changes in actual allocations, DSAs could "perceive" them in a relatively quick fashion, thus providing estimated allocations that comply with such behaviors.

This type of exercise is important to check the quality of how the proposed state space models (linear and non-linear) "would work" in practical situations in which the management style has to be determined over time; this is relevant, since, very rarely would a real investment fund have such an aggressive behavior as the one presented by artificial portfolios; and, anyway, there was a satisfactory replication of these portfolios over time.

\section{Analysis of Actual Portfolios}

In the present section, DSA models will be applied to two Brazilian investment funds: a stock fund and a US Dollar exchange rate fund.

\subsection{First exercise: stock fund}

In the first exercise, we estimate the allocations/exposures for the Index FIA fund, classified by the Brazilian National Association of Investment and Development Banks (ANBID) as an indexed Ibovespa stock exchange fund. We then present the graphs of the behavior of estimated allocations for each type of DSA. The period to be considered goes from the second week in January 2001 to the last week in December 2002; therefore, we have 103 observations for the analysis. Table 2 displays the mean squared error and pseudo- $R^{2}$ for each analysis. ${ }^{7}$

The tables in Appendix A show some aspects on the estimations, such as computational time and some diagnoses based on standardized (pseudo-)innovations, which indicate relatively reasonable adjustments. It should be borne in mind that there are no theoretical guarantees that the normality assumption should be satisfied for the adjustment of the model to a strong DSA - see further details in Pizzinga (2004).

It should also be pointed out that innovation analysis for such models did not reveal dependency structures in the second conditional moment of the return. ${ }^{8}$ This justifies the absence of GARCH type error terms on the models proposed here. To understand the reasons for this, we should first note that GARCH effects are either nonexistent or weak for low frequency financial data, such as weekly data. Secondly, the models here entertained, by including return series of asset class indexes as regressors (equations (19), (20) and (21)), already control for this type of effect.

\footnotetext{
${ }^{7}$ Pseudo- $R^{2}$ is given by the squared correlation between the fund return and its value predicted by the model.

${ }^{8}$ Box-Ljung tests were carried out for the square of standardized innovations and the BDS test was used for the independence of stardardized innovations.
} 

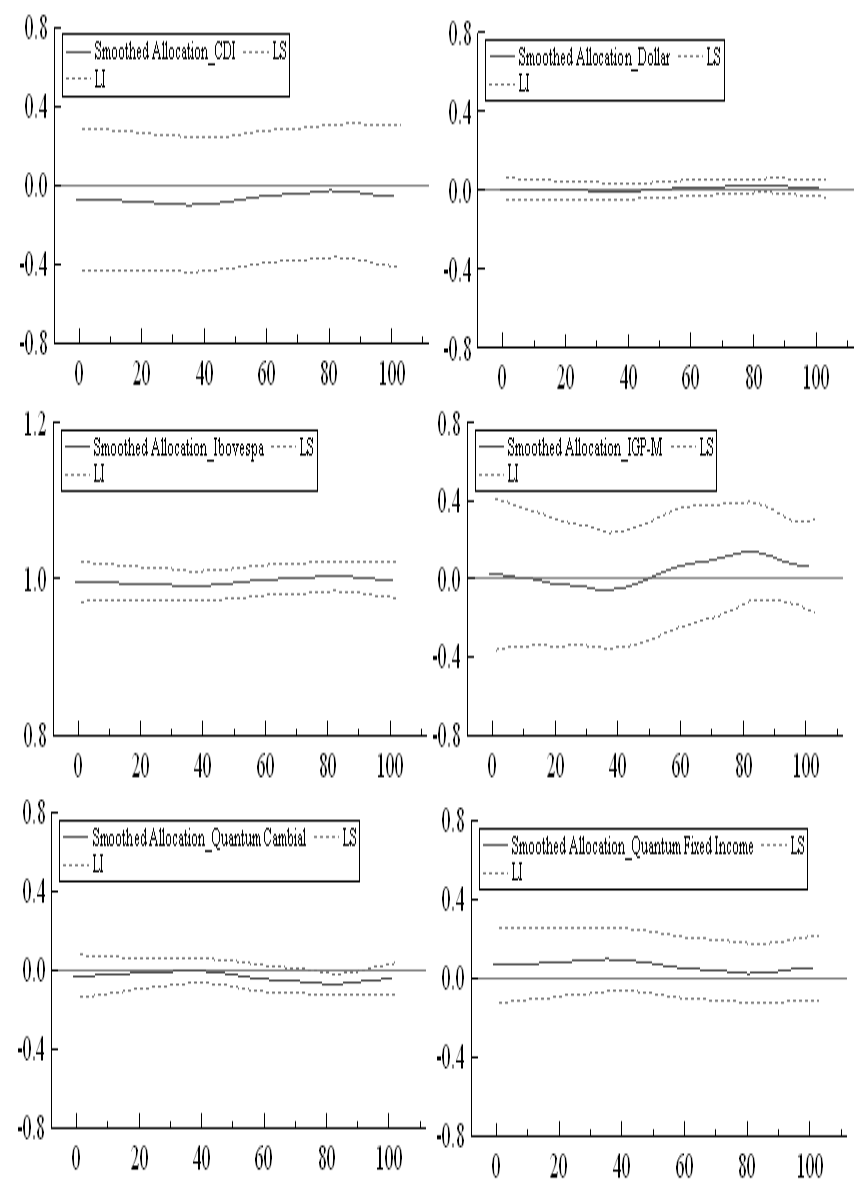

Figure 6

Weak dynamic style analysis of the Itaú index FIA fund 

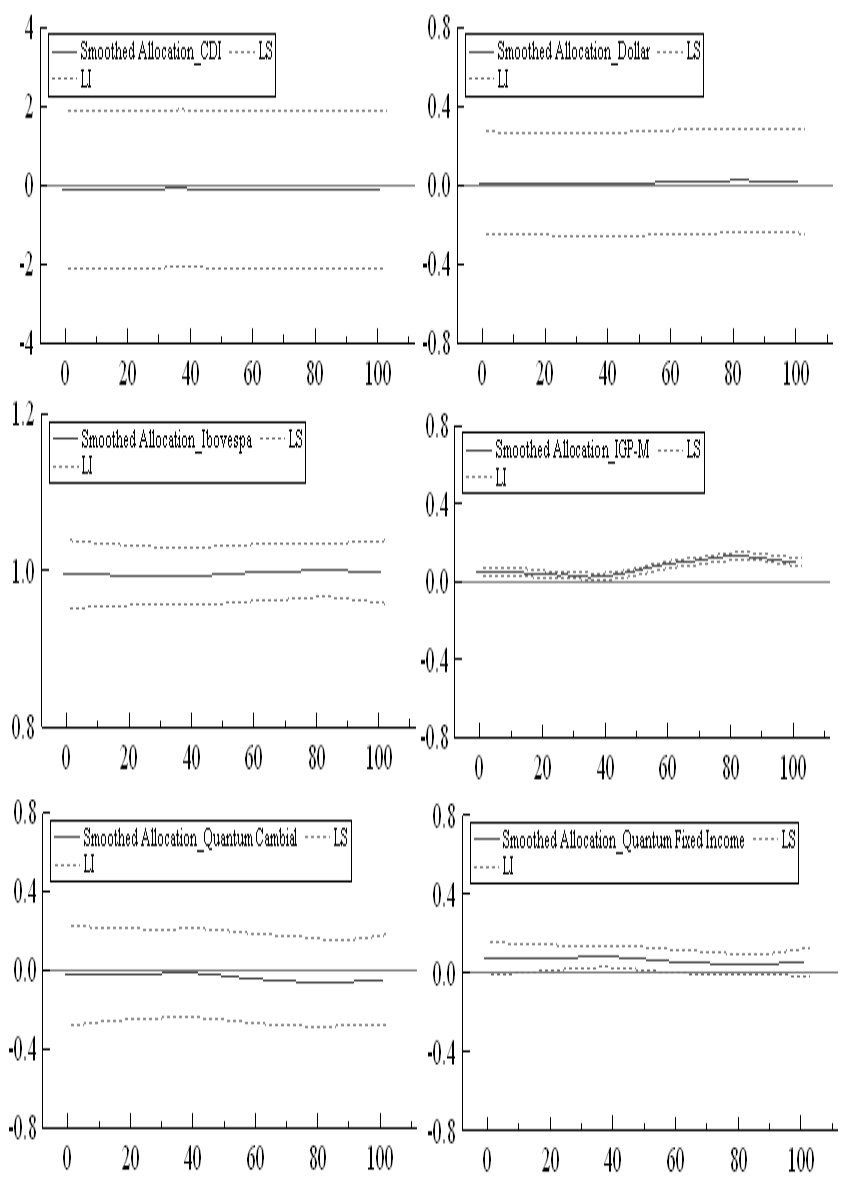

Figure 7

Semi-strong dynamic style analysis of the Itaú index FIA fund 

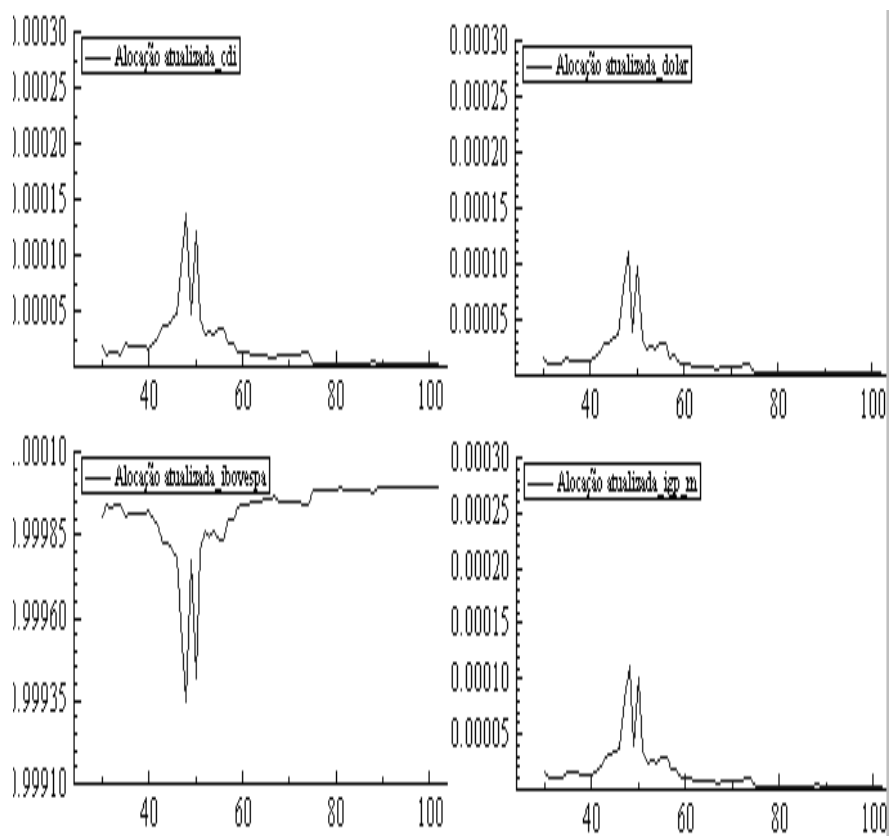

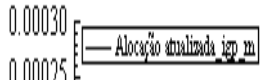
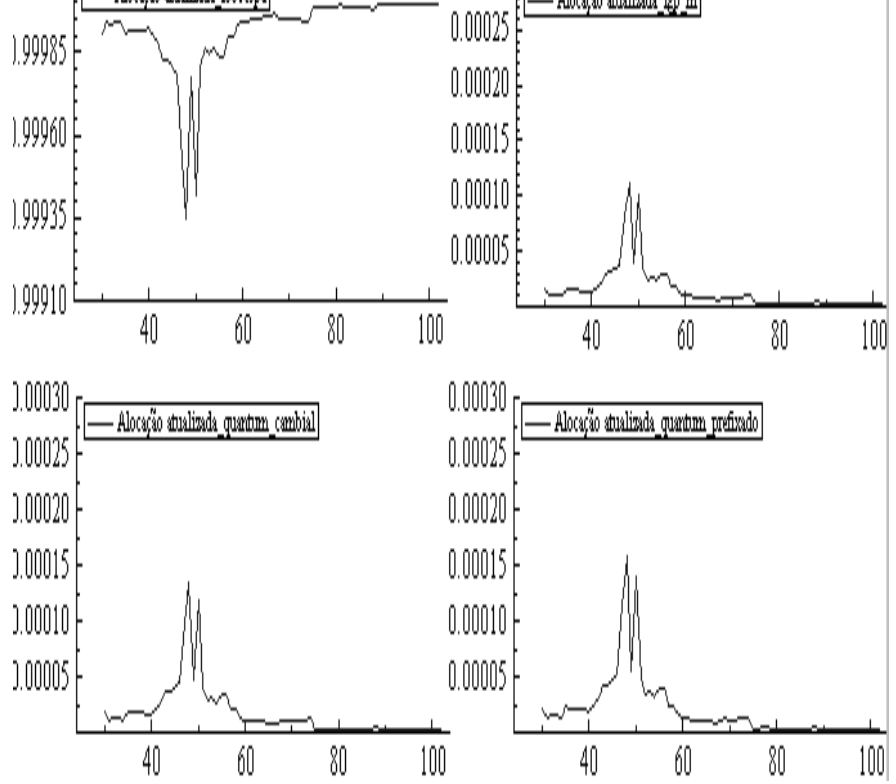

Figure 8

Strong dynamic style analysis of the Itaú index FIA fund using the extended Kalman filter 

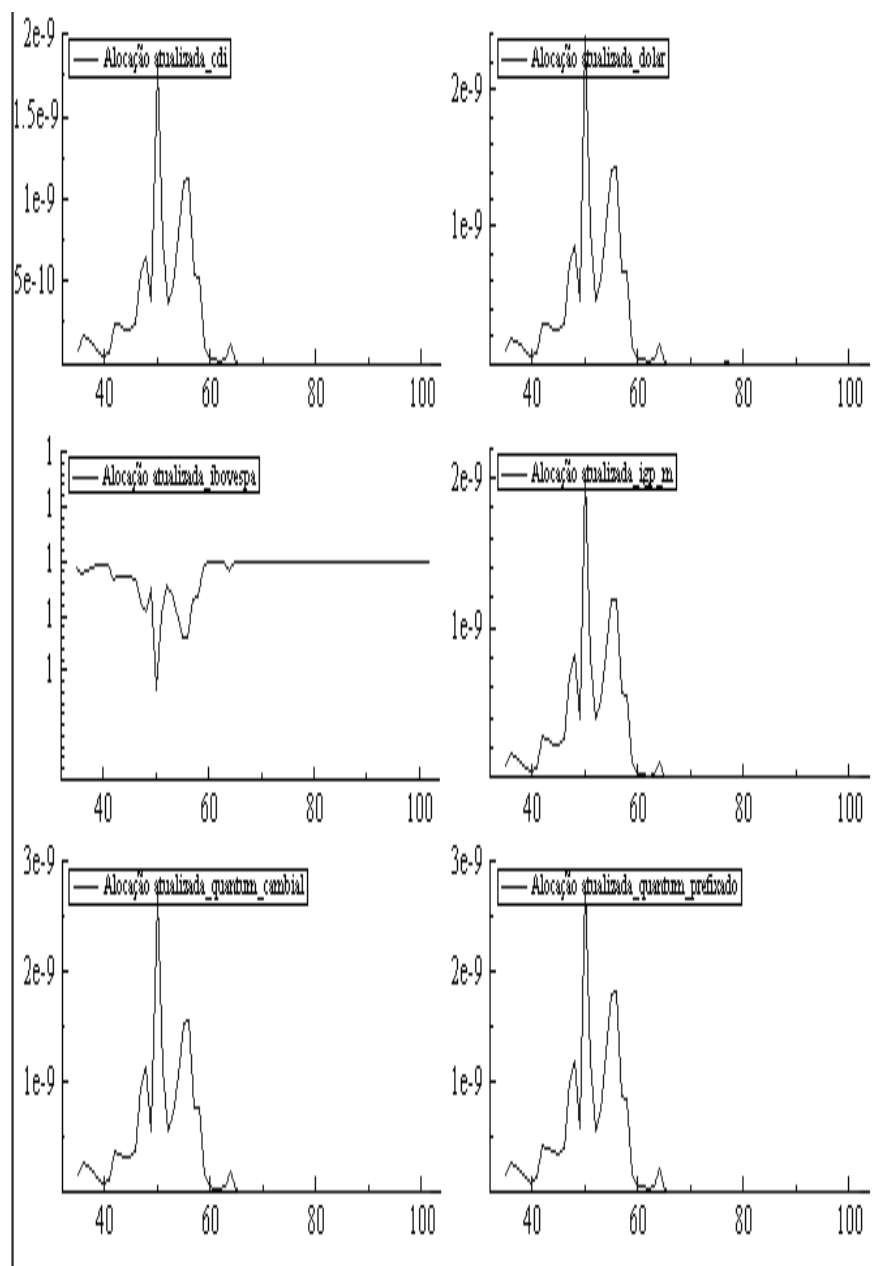

Figure 9

Strong dynamic style analysis of the Itaú index FIA fund using the Monte Carlo simulation filter 
Table 2

Adherence measures associated with dynamic style analysis of the Itaú index FIA fund

\begin{tabular}{l|c|c}
\hline \multicolumn{1}{c|}{ DSA } & Mean square error & Pseudo- $R^{2}$ \\
\hline Weak & 0.169 & 0.994658 \\
Semi-strong & 0.117 & 0.995228 \\
Strong using the extended Kalman filter & 0.078 & 0.996753 \\
Strong using the Monte Carlo simulation filter & 0.084 & 0.996523 \\
\hline
\end{tabular}

According to figures 6,7,8 and 9, and to table 2, one can observe that all four DSAs confirmed the fund-indexed management policy, since allocations to the stock market - effectively represented by IBOVESPA - were extremely close to 1 (one) along the whole period of analysis. With some exceptions (see, for instance, allocations to the fixed-income market represented by CDI in weak and semi-strong DSAs), the behavior of the fund indicates that no operations occurred outside the stock market. According to the adherence measures, the semi-strong DSA showed slightly better results than the weak DSA, thus corroborating the validity of portfolio constraint for this fund. In their turn, strong DSAs outperformed semi-strong DSA, which may be seen as a sign that short sales restrictions also seem to be supported by the data.

\subsection{Second exercise: US Dollar exchange rate fund}

We now analyze the performance of DSA models when applied to the HSBC FIF Cambial fund, which, according to ANBID, belongs to the Dollar Referenced Funds category. This category theoretically includes investment funds whose target is to have profitability close to that offered by the Dollar exchange rate market (spot market and/or derivatives market), being subject to the oscillations in the fixed-income market. Therefore, as the same six indicators from the previous studies were used, stronger estimated allocations are a priori expected for the Dollar and/or Quantum Cambial, with occasional movements into CDI and Quantum Fixed Income. The period of analysis goes from the first week of 2001 to the last week of 2002, totaling 104 observations.

The estimated allocations for this exchange rate fund produced by the DSA models are depicted in the next figures. These together with the goodness of fit measures displayed in table 3 and in the tables in Appendix B suggest that, overall, the DSA models perform pretty adequately. As before, we use the same kind of arguments used in the previous exercise to justify the absence of GARCH terms in the DSA models used in the present context. 

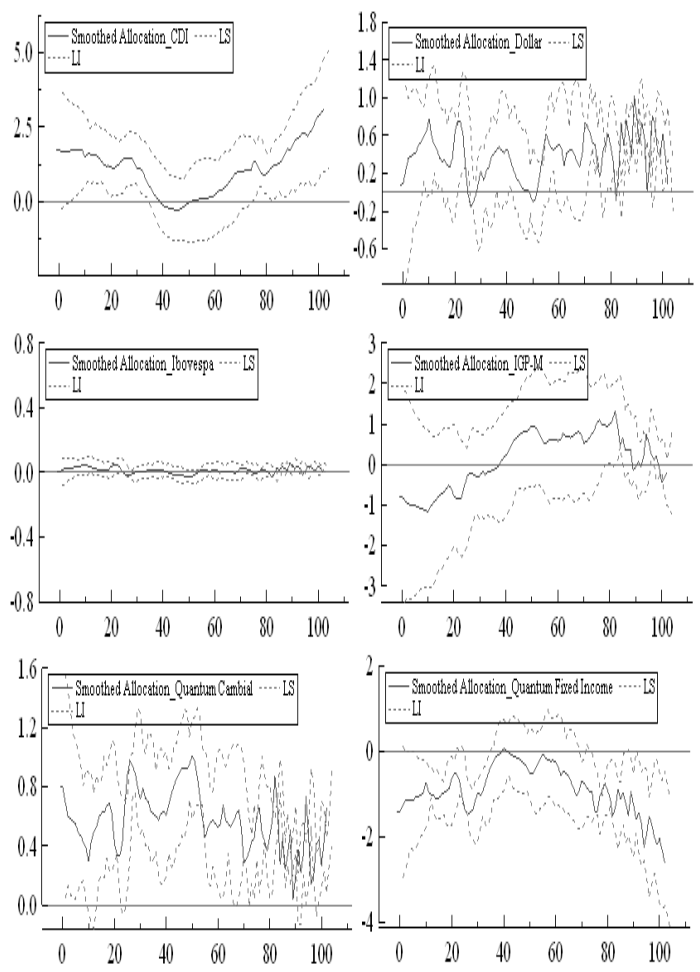

Figure 10

Weak dynamic style analysis of the HSBC FIF cambial fund 

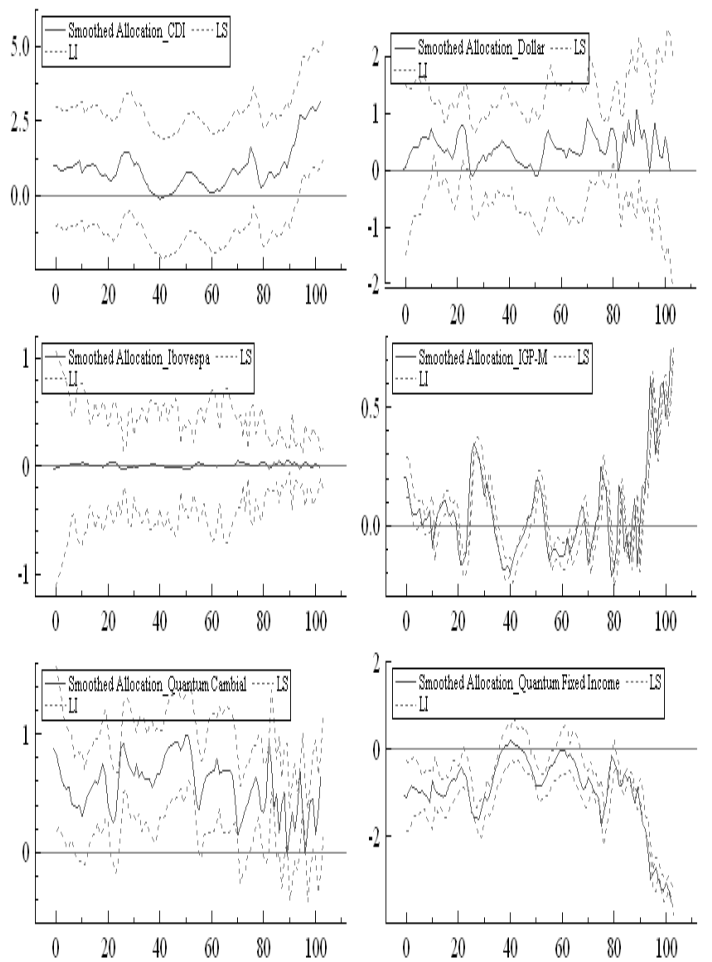

Figure 11

Semi-strong dynamic style analysis of the HSBC FIF cambial fund 

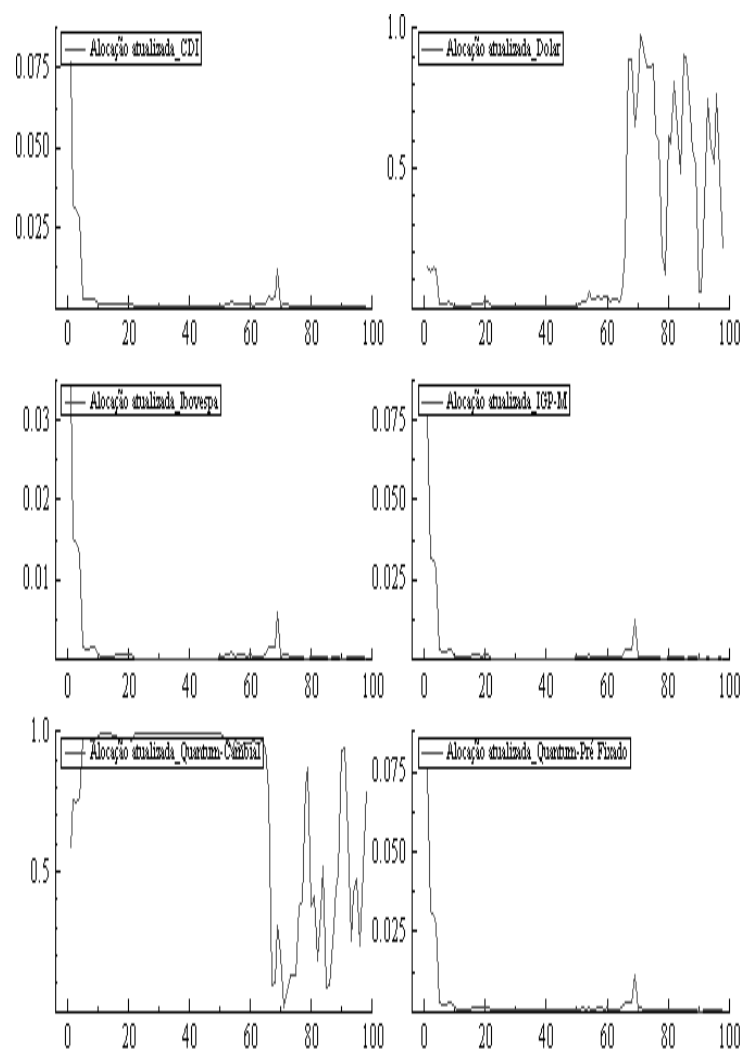

Figure 12

Strong dynamic style analysis of the HSBC FIF cambial fund using the extended Kalman filter 

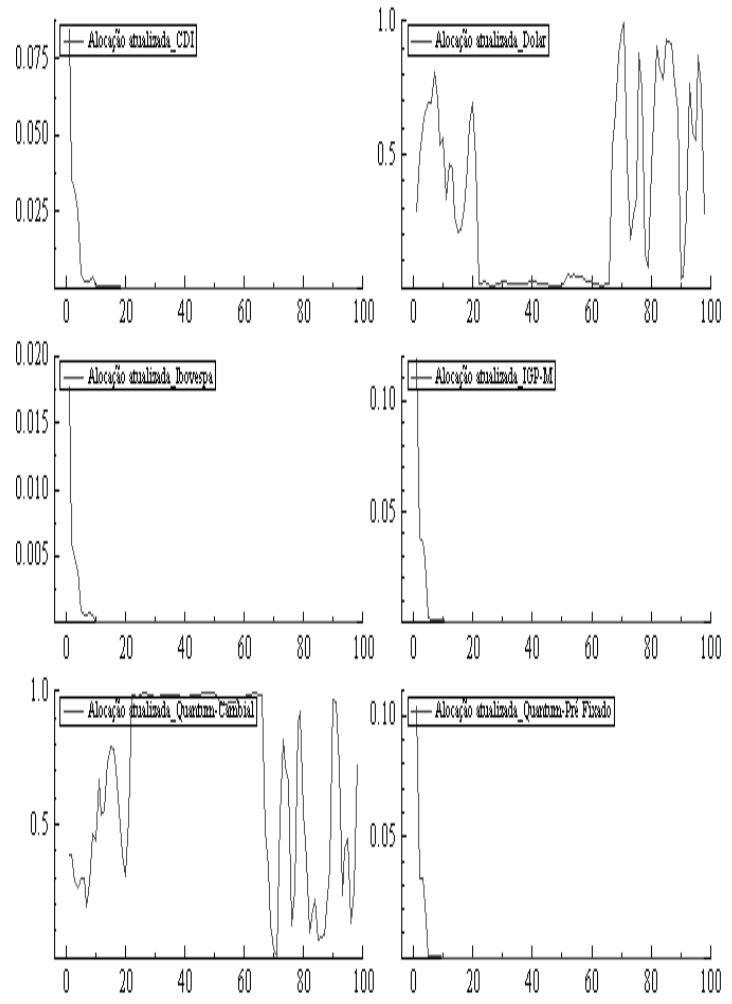

Figure 13

Strong dynamic style analysis of the HSBC FIF cambial fund using the Monte Carlo simulation filter 
Table 3

Adherence measures associated with dynamic style analysis of the HSBC FIF cambial fund

\begin{tabular}{l|c|c}
\hline \multicolumn{1}{c|}{ DSA } & Mean square error & Pseudo-R2 \\
\hline Weak & 1,007 & 0,88134 \\
Semi-strong & 0,855 & 0,90052 \\
Strong using the extended Kalman filter & 0,981 & 0,87709 \\
Strong using the Monte Carlo simulation filter & 1,089 & 0,87224 \\
\hline
\end{tabular}

Figures 10, 11, 12 and 13, and table 3 show that all four analyses provide sound evidence that the fund was well-positioned in the exchange derivatives market - represented by the Quantum Cambial - up to the $60^{t h}$ week (second week of February 2002), after which there were constant oscillations between this market and the spot market - represented by the Dollar. Also note that, according to weak and semi-strong DSAs, there is strong evidence of sold positions in some moments of the fixed-income derivatives market - represented by the preset Quantum -, especially in the last weeks of 2002. Obviously, such perspective cannot be constructed based on the strong DSA, due to the positivity constraints of the estimated allocations. According to adherence measures, semi-strong DSA proved the most appropriate for the fund in question. Moreover, the worst results regarding these measures for strong DSAs can be interpreted alongside the signs of occasional sold positions in weak and semi-strong DSAs, indicating that the non-linear specification given by equations (18) and (21) is not appropriate for analyzing the dynamics of allocations to this fund.

\subsection{Static style analyses}

For the sake of comparison, we present the strong SSAs for the previously analyzed two funds. The analyzed periods were the same as those in DSAs, and the implementation was made using the Quantum-Axis system. ${ }^{9}$

Table 4

Strong static style analysis of the Itaú Index FIA fund

\begin{tabular}{l|c}
\hline \multicolumn{1}{c|}{ Asset class index } & Estimated allocation \\
\hline CDI & 0 \\
Dollar & 0 \\
Ibovespa & 1 \\
IGP-M & 0 \\
Quantum Cambial & 0 \\
Quantum Fixed Income & 0 \\
\hline
\end{tabular}

\footnotetext{
${ }^{9}$ www.quantumfundos.com.br.
} 
Table 5

Strong static style analysis of the HSBC FIF cambial fund

\begin{tabular}{l|c}
\hline \multicolumn{1}{c|}{ Asset class index } & Estimated allocation \\
\hline CDI & 0 \\
Dollar & 0,52 \\
Ibovespa & 0 \\
IGP-M & 0 \\
Quantum Cambial & 0,48 \\
Quantum Fixed Income & 0 \\
\hline
\end{tabular}

The information contained in Tables 3 and 4 provides evidence, by static analysis, that the funds are concentrated in correct markets. However, the same criticism prevails: SSA is unable to spot effective and dynamic changes in the composition of the fund portfolio, over time. This behavior can be observed through the results obtained from DSAs using the HSBC FIF Cambial portfolio (subsection 6.2, figures 10,11, 12 and 13). The most evident change is the one observed in the allocations in exchange rate markets from the $60^{\text {th }}$ week, which was described in detail in subsection 6.2 .

\section{Conclusions}

This article presents a framework for the implementation of dynamic style analysis (DSA) using linear and non-linear state space models with linear restrictions on the state vector. Our examples illustrate some of the ways by which these techniques can improve upon standard static style analysis, but there are other practical situations for which we believe DSA may also be useful. For instance, it may be used by either managers of pension funds or managers of funds of funds in order to estimate the actual allocations of a fund for which the pension fund/fund of funds has acquired quotas, and to check whether these estimates, whenever taken as good proxies for the true exposures, agreed with those written in the contracts between these parties. It is also a helpful device for companies involved in fund rating, since DSA provides an effective way of estimating the actual allocations of a fund of asset management companies, and this is an important input in the process of working out fund ratings. Finally, DSA as proposed in this article can also be used by a fund's head manager for monitoring a subordinated manager's style.

The techniques presented here can be used in different settings from the ones exemplified here. For example, they can consider indicators for funds and foreign markets, and can also deal with other types of data frequencies, such as daily or monthly. On the modeling side, one could propose a stochastic process for the exposures other than the random walk. For instance, it may be adequate to extend the models proposed here to tackle switching regimes in allocations. A useful and revealing exercise that could help to establish the efficacy of our technique and provide information to new specifications of the state equation would be to compare, for a real investment fund, the allocations recovered by DSA with the observed and true allocations. 


\section{Appendix A}

\section{Information on the Adjustment of the Models Proposed for the Itaú} Index FIA Fund

Table A.1

Analytical results for the weak dynamic style analysis of the Itaú index FIA fund

\begin{tabular}{l|c}
\hline \multicolumn{1}{c|}{ Attribute } & Value \\
\hline Log-likelihood & $-26,335$ \\
Number of iterations & 52 \\
Computational time (approx.) & 3 seconds \\
Ljung-Box test (30 lags) & $44,846$ (p-value $=0,040)$ \\
Jarque-Bera test & $2,325$ (p-value $=0,313)$ \\
\hline
\end{tabular}

Table A.2

Analytical results for the semi-strong dynamic style analysis of the Itaú index FIA fund

\begin{tabular}{l|c}
\hline \multicolumn{1}{c|}{ Attribute } & Value \\
\hline Log-likelihood & 348,516 \\
Number of Iterations & 70 \\
Computational time (approx.) & 4 seconds \\
Ljung-Box test (30 lags) & $52,230$ (p-value $=0,007)$ \\
Jarque-Bera test & $0,088$ (p-value $=0,957)$ \\
\hline
\end{tabular}

Table A.3

Analytical results for the strong dynamic style analysis of the Ita ú Index FIA fund using the extended Kalman filter

\begin{tabular}{l|c}
\hline \multicolumn{1}{c|}{ Attribute } & Value \\
\hline Approximate log-likelihood & $-25,948$ \\
Number of iterations & 17 \\
Computational time (approx.) & 9 seconds \\
Ljung-Box test(30 lags) & $48,078$ (p-value $=0,02)$ \\
Jarque-Bera test & $15,986$ (p-value $=0,000)$ \\
\hline
\end{tabular}

Table A.4

Analytical results for the strong dynamic style analysis of the Itaú Index FIA fund using the Monte Carlo simulation filter.

\begin{tabular}{l|c}
\hline \multicolumn{1}{c|}{ Attribute } & Value \\
\hline Approximate log-likelihood & $-31,797$ \\
Number of iterations & 14 \\
Number of "draws" (K) & 600 \\
Computational time (approx.) & 6 minutes \\
Ljung-Box test (30 lags) & $62,019$ (p-value $=0,001)$ \\
Jarque-Bera test & $175,150$ (p-value $=0,000)$ \\
\hline
\end{tabular}




\section{Appendix B}

\section{Information on the Adjustment of the Models Proposed for the HSBC} FIF Cambial Fund

Table B.1

Analytical results for the weak dynamic style analysis of the HSBC FIF cambial fund

\begin{tabular}{l|c}
\hline \multicolumn{1}{c|}{ Attribute } & Value \\
\hline Log-likelihood & $-87,942$ \\
Number of iterations & 66 \\
Computational time (approx.) & 3 seconds \\
Ljung-Box test (30 lags) & $22,725$ (p-value $=0,826)$ \\
Jarque-Bera test & $0,279$ (p-value $=0,870)$ \\
\hline
\end{tabular}

Table B.2

Analytical results for the semi-strong dynamic style analysis of the HSBC FIF cambial fund

\begin{tabular}{l|c}
\hline \multicolumn{1}{c|}{ Attribute } & Value \\
\hline Log-likelihood & 405,809 \\
Number of iterations & 68 \\
Computational time (approx.) & 3 seconds \\
Ljung-Box test (30 lags) & $21,852(\mathrm{p}$-value $=0,859)$ \\
Jarque-Bera test & $1,646(\mathrm{p}$-value $=0,439)$ \\
\hline
\end{tabular}

Table B.3

Analytical results for the strong dynamic style analysis of the HSBC FIF cambial fund using the extended Kalman filter

\begin{tabular}{l|c}
\hline \multicolumn{1}{c|}{ Attribute } & Value \\
\hline Approximate likelihood & $-131,93$ \\
Number of iterations & 41 \\
Computational time (approx.) & 20 seconds \\
Ljung-Box test (30 lags) & $26,790$ (p-value $=0,634)$ \\
Jarque-Bera test & $64,191$ (p-value $=0,000)$ \\
\hline
\end{tabular}

Table B.4

Analytical results for the strong dynamic style analysis of the HSBC FIF Cambial fund using the Monte Carlo simulation filter

\begin{tabular}{l|c}
\hline \multicolumn{1}{c|}{ Attribute } & Value \\
\hline Approximate log-likelihood & $-124,092$ \\
Number of iterations & 62 \\
Number of "draws" (K) & 600 \\
Computational time (approx.) & 22 minutes \\
Ljung-Box test (30 lags) & $29,709$ (p-value $=0,481)$ \\
Jarque-Bera test & $17,906$ (p-value $=0,000)$ \\
\hline
\end{tabular}




\section{References}

Anderson, B. D. O. \& Moore, J. B. (1979). Optimal Filtering. Prentice Hall.

Brockwell, P. J. \& Davis, R. A. (1996). Introduction to Time Series and Forecasting. Springer Texts in Statistics.

Brown, S. J. \& Goetzmann, W. N. (2003). Hedge funds with style. The Journal of Portfolio Management, Winter:101-112.

De Jong, P. (1989). Smoothing and interpolation with the state-space model. Journal of the American Statistical Association, 84:1085-1098.

De Roon, F. A., Nijman, T. E., \& Ter Horst, J. R. (2004). Evaluating style analysis. Journal of Empirical Finance, 11(1):29-53.

Doornick, J. A. (2001). Ox 3.0: An Object-Oriented Matrix Programming Language. Timberlake Consultants Ltd.

Doran, H. E. (1992). Constraining Kalman filter and smoothing estimates to satisfy time-varying restrictions. Review of Economics and Statistics, 74:568-572.

Doucet, A., Freitas, N., \& Gordon, N. (2001). Sequential Monte Carlo Methods in Practice. Springer.

Durbin, J. \& Koopman, S. J. (2001). Time Series Analysis by State Space Methods. Oxford Statistical Science Series.

Fuhrer, J. C. (1992). Inferring changes in expectation behavior over time: An application of nonlinear time-varying parameters estimation. Journal of Business \& Economic Statistics, 10:169-177.

Gamerman, D. (1998). Markov chain Monte Carlo for dynamic generalized linear models. Biometrika, 85:215-227.

Harvey, A. C. (1989). Forecasting, Structural Time Series Models and the Kalman Filter. Cambridge University Press.

Koopman, S. J., Shephard, N., \& Doornik, J. A. (2002). SsfPack 3.0 beta02: Statistical algorithms for models in state space. Unpublished paper. Department of Econometrics, Free University, Amsterdam.

Otten, R. \& Bams, D. (2000). Statistical tests for return-based style analysis. http://www.fdewb.unimaas.nl/finance/faculty/bams/stattests.pdf.

Pizzinga, A. (2004). Modelos em espaço de estado com restrições nas componentes de interesse: Aplicações em análise dinâmica de estilo para fundos de investimento brasileiros. Master's thesis, Department of Electrical Engineering - Catholic University of Rio de Janeiro. 
Pizzinga, A., Fernandes, C., \& Contreras, S. (2006a). Restricted Kalman filtering revisited. Paper submitted for publication. Department of Electrical Engineering - Catholic University of Rio de Janeiro.

Pizzinga, A., Fernandes, C., \& Junger, W. (2006b). Nonlinear state space methods for the estimation of a dynamic asset class factor model. Paper submitted for publication. Department of Electrical Engineering - Catholic University of Rio de Janeiro.

Sharpe, W. F. (1988). Determining a fund's effective asset mix. Investment Management Review, pages 59-69.

Sharpe, W. F. (1992). Asset allocation: Management style and performance measurement. The Journal of Portfolio Management, Winter:7-19.

Shephard, N. (1994). Partial non-Gaussian state space. Biometrika, 81:115-131.

Shephard, N. \& Pitt, M. (1997). Likelihood analysis of non-Gaussian measurement time series. Biometrika, 84:653-667.

Swinkels, L. \& Van de Sluis, P. J. (2001). Return-based style analysis with timevarying exposures. http://center.uvt.nl/phd-stud/swinkels/research.html.

Tanizaki, H. (1996). Nonlinear Filters. Springer, 2nd. edition.

Tanizaki, H. \& Mariano, R. (1996). Nonlinear filters based on Taylor series expansions. Communications in Statistics, 5(6).

Varga, G. \& Valli, M. (1998). Análise de estilo baseada no retorno. Revista da ANBID. 